\title{
Efecto de un programa de capacitación del sistema de evaluación SOFIT en estudiantes de Licenciatura en Actividad Física y Deporte sobre la intensidad y contexto de la clase de Educación Física
}

Javier A. Hall-López', Paulina Y. Ochoa-Martínez', Luis Roberto Monreal Ortiz² y Bartolomé J. Almagro 3

\author{
'Facultad de Deportes, Universidad Autónoma de Baja California (UABC), \\ México \\ ${ }^{2}$ Facultad de Educación Física y Deporte de la Universidad Autónoma de \\ Sinaloa, México. \\ ${ }^{3}$ Facultad de Educación, Psicología y Ciencias del Deporte de la \\ Universidad de Huelva, España \\ Email: javierhall@uabc.edu.mx
}

\begin{abstract}
RESUMEN: El objetivo principal de la investigación fue evaluar la intensidad y contexto de la clase de Educación Física (EF) antes y después de una capacitación a estudiantes de Licenciatura en Actividad Física y Deporte. Para ello, se empleó un diseño cuasi-experimental pre-post con un solo grupo. Los sujetos participantes en la capacitación fueron 64 estudiantes de la Licenciatura en Actividad Física y Deporte, de la Escuela de Deportes de la Universidad Autónoma de Baja California (México), que cursaban la asignatura de Evaluación de la Educación Física. A estos estudiantes se les pidió diseñar y aplicar tres clases de EF antes de la capacitación y tres clases de EF después de la capacitación, realizándose la evaluación diagnóstica y sumativa respectivamente. Para hacer esta comparación se diseñó un curso de capacitación cuyos contenidos estuvieron enfocados en el objetivo, validación, descripción y sistema metodológico de registro del sistema SOFIT. Los resultados mostraron la mejora de los docentes después de la realización del curso, ya que la intensidad de la actividad física en sus clases aumentó, también lo hizo el índice de actividad física moderada a vigorosa de su alumnado, que pasó de una $37,3 \%$ en el pretest a un $70.4 \%$ en el postest. Con respecto al contexto, se disminuyó el tiempo empleado para administrar y organizar las actividades, aprovechándose más el tiempo de clase para el acondicionamiento físico, el desarrollo de habilidades y juegos. Se concluye que el curso y el uso del SOFIT ayudaron a los estudiantes a maximizar el uso del tiempo de la clase de EF.
\end{abstract}

PALABRAS CLAVE: Enseñanza y formación, enseñanza superior, intervención, tiempo de compromiso motor. 


\title{
Effect of a training program of the SOFIT evaluation system on Physical Activity and Sports students on the intensity and context of the Physical Education class
}

\begin{abstract}
The main objective of the research was to evaluate the intensity and context of the Physical Education (PE) class before and after of a training for Physical Activity and Sports students. For this, a quasi-experimental pre-post design with a single group was used. The subjects participating in the training were 64 Physical Activity and Sports student, of the School of Sports of the Autonomous University of Baja California (Mexico), who were studying the subject of Physical Education Evaluation. These students were asked to design and apply three classes of PE before training and three classes of PE after training, performing the diagnostic and summative evaluation respectively. To make this comparison, a training course was designed whose contents were focused on the objective, validation, description and methodological registration system of the SOFIT system. The results showed the improvement of teachers after the completion of the course, since the intensity of physical activity in their classes increased, so did the index of moderate to vigorous physical activity of their students, which went from $37.3 \%$ in the pretest to $70.4 \%$ in the posttest. With respect to the context, the time taken to manage and organize the activities was reduced, taking advantage of class time for physical conditioning, skills development and games. It is concluded that the course and the use of SOFIT helped students maximize the use of the PE class time.
\end{abstract}

KEYWORDS: Teaching and training, higher education, intervention, engagement motor time

Este artículo está publicado como capítulo en el libro: Hall, J. A., Ochoa, P. Y., y SáenzLópez, P. (2018). Intensidad, salud, motivación y adherencia en Educación Física. Huelva: Servicio de publicaciones de la Universidad de Huelva.

\section{INTRODUCCIÓN}

Los resultados de la última Encuesta Nacional de Salud y Nutrición (ENSANUT; Olaiz et al., 2006) en México, reportan que la obesidad en escolares de 5 a 11 años de edad, aumentó de 5.3\% a 9.4\% en niños y de 5.9\% a 8.7\% en niñas de 1999 al 2006 mostrando con ello una prevalencia combinada de sobrepeso y obesidad en aumento, pasando de $18.6 \%$ a $26.3 \%$.

Aunque la obesidad tiene un origen multifactorial, los cambios en el estilo de vida en los últimos años han incluido la adopción de alimentos altos en energía (densos en los contenidos de grasa e hidratos de carbono), así como una disminución de la actividad física, estos factores provocan un desequilibrio en el balance energético hacia la acumulación de kilocalorías y como consecuencia el desarrollo de obesidad en todas las edades alrededor del mundo (Ebbeling et al., 2002; Hernández et al., 1999; Trost et al., 2001). 
Debido a que la actividad física es el único componente de gasto energético que puede ser modificado de manera voluntaria, estudiar este componente es importante en la investigación orientada a la prevención y tratamiento de la obesidad, adicionalmente la actividad física tiene diversos beneficios en edad infantil como la mejora de las capacidad física condicionales y coordinativas (Trost et al., 2001), la disminución de riesgo cardiovascular (Freedman et al., 1999) incrementa la autoestima, reduce la depresión y mejora la calidad de vida (Ebbeling et al., 2002).

La educación básica, dada su amplia cobertura, es un medio ideal para la promoción de la actividad física y para desarrollar aptitudes positivas en hábitos saludables, que servirá de base para que los niños tiendan a adoptar un estilo de vida saludable como adultos, y que a futuro prevengan la morbilidad y mortalidad de enfermedades relacionadas con la obesidad, reduciendo los altos costos en sus tratamientos (Luepker et al., 1996; Nader et al., 1999). En México se han realizado investigaciones descriptivas utilizando el sistema para observar el tiempo de instrucción de actividad física (SOFIT). Los resultados mostraron una duración de la clase por debajo de los 50 minutos oficiales, una intensidad moderada a vigorosa por debajo del $50 \%$ de acuerdo a los estándares internacionales como la United States National Association for Sport and Physical Education (NASPE) y un contexto en el cual el administrar la clase y el desarrollo de habilidades motrices conlleva gran parte de la totalidad de la clase. Se percibió una gran cantidad de tiempo en que los alumnos permanecieron parados mientras el profesor organizaba al grupo para participar, la falta de material didáctico para tener más oportunidad de participar, las largas filas para tener la oportunidad de participar y los tiempos de transición entre las actividades. Concluyendo que en la clase de educación física no se promueve la actividad física moderada a vigorosa y se requiere revisar la manera de impartirla.

Por lo anterior, el propósito de la investigación fue evaluar la intensidad y contexto de la clase de educación física (en adelante EF) antes y después de una capacitación a estudiantes de Licenciatura en Actividad Física y Deporte de la Facultad de Deportes de la Universidad Autónoma de Baja California.

\section{MÉTODO}

La investigación fue llevada a cabo a través de un diseño cuasi-experimental prepost con un solo grupo (clasificación de Montero y León, 2002). Se empleó un muestreo no probabilístico por conveniencia, realizando una evaluación diagnostica; un protocolo de intervención mediante capacitación con contenidos del sistema para observar el tiempo de instrucción de actividad física (SOFIT) y evaluación sumativa. Se presentó un diseño que permitiera responder a la pregunta de: en qué medida una capacitación de cuatro horas en contenidos relacionados con sistema para observar el tiempo de instrucción de actividad física (SOFIT) puede modificar el diseño de una clase de EF en cuanto a la intensidad, contexto, índice de actividad física moderada a vigorosa y frecuencia cardiaca promedio, al aplicarla entre estudiantes de Licenciatura en Actividad Física y Deportes.

\subsection{Participantes}

Los sujetos participantes en la capacitación fueron 64 estudiantes de dos grupos de séptimo semestre de la Licenciatura en Actividad Física y Deporte, de la Escuela de Deportes de la Universidad Autónoma de Baja California (México), que cursaban la asignatura de Evaluación de la Educación Física. A estos estudiantes se les pidió diseñar y aplicar tres clases de EF antes de la capacitación y tres clases de EF después 
de la capacitación, realizándose la evaluación diagnóstica y sumativa respectivamente. Para hacer esta comparación se diseñó un curso de capacitación cuyos contenidos estuvieron enfocados en el objetivo, validación, descripción y sistema metodológico de registro del sistema SOFIT.

\subsection{Instrumentos}

La evaluación diagnóstica y sumativa de las variables dependientes se realizó a través del System for Observing Fitness and Instruction Time (McKenzie, Sallis, y Nader, 1991), que es un sistema para observar el tiempo de instrucción de actividad física (SOFIT por sus siglas en inglés). Se evaluaron seis clases de EF orientadas para edades de 9 a 11 años, eligiendo para el tiempo de instrucción a 4 estudiantes de cada clase para observarlos en secuencia rotatoria, durante 12 intervalos de 20 segundos cada uno, repitiéndose las observaciones durante toda la clase, siguiendo el audio de SOFIT (McKenzie et al., 1991) en un reproductor MP3 Samsung YP-U6AB. En la evaluación SOFIT se usaron códigos para clasificar los niveles de actividad, los cuales permitieron estimar la energía gastada asociada con la actividad física. Este procedimiento se ha usado para evaluar los efectos del entrenamiento relacionado con el currículum de EF. Los códigos se clasifican en cuatro: 1) acostado, 2) sentado, 3) parado, 4) caminando, y 5) muy activo, que corresponde a correr o a cuando el estudiante realiza más actividad física que la que corresponde al caminar ordinario. Estos códigos han sido calibrados monitoreando los latidos del corazón y el sistema ha sido validado usando acelerómetros Caltrac (McKenzie, Sallis, y Armstrong, 1994). El índice de actividad física moderada a vigorosa se determino sumando porcentualmente los códigos: 4) caminando, y 5) muy activo del total del tiempo de la clase.

Un segundo aspecto que evalúa el SOFIT simultáneamente con la actividad física del estudiante es el contexto de la clase. Este contexto se codifica en siete categorías, $\mathrm{M}=$ Contenido general, $\mathrm{P}=$ Conocimiento específico, $\mathrm{K}=$ Conocimiento general, $\mathrm{F}=$ Acondicionamiento físico, $\mathrm{S}=$ Desarrollo de habilidades, $\mathrm{G}=$ Juego, $\mathrm{O}=$ Otros.

El tiempo de la clase de EF se cronometró con un Casio HS3W, estableciendo el inicio y fin de la clase de EF de acuerdo al horario establecido para la sesión de 50 minutos.

La frecuencia cardiaca promedio fue determinada a través de un monitor de frecuencia cardiaca (de la marca Polar), tomándolo durante la clase a 4 alumnos en cada sesión.

\subsection{Procedimiento}

Se solicitaron los permisos necesarios (tanto a la institución donde se desarrolló dicho estudio como al alumnado que participó en él) y se informó a los estudiantes universitarios que la participación en el estudio era totalmente voluntaria.

\subsection{Análisis de los datos}

Para el análisis de los datos se utilizó el programa SPSS Versión 23.0, donde se realizaron cálculos para su procesamiento estadístico, obteniendo de manera descriptiva la media y la desviación típica. Además, con los resultados obtenidos se realizaron gráficas en el software Excel de Windows que reflejan los resultados más significativos de esta investigación. 


\section{RESULTADOS}

En la Figura 1 se muestra la distribución porcentual de la intensidad de actividad física evaluada antes y después de la capacitación. La Figura 2 muestra un índice de actividad física moderada a vigorosa diagnostico de $37.3 \%$ y sumativo de $70.4 \%$ del total de la clase el cual después de la capacitación cumple con los estándares establecidos por el United States' National Association for Sport and Physical Education (NASPE).

En la Figura 3 se refleja el contexto de la clase derivado de la planeación de la clase de EF donde se destaca en mayor medida al apartado M (Contenido general). Se utilizó el tiempo de clase en contenidos que incluyen la transición, administración y descanso antes de la capacitación; disminuyendo después de ésta y dedicando más tiempo de la clase de EF. El mismo patrón ocurrió en realizar actividades de desarrollo de habilidades y lo contrario en actividades que involucran acondicionamiento físico, quedando el juego como parte del contexto de la clase que se utilizó en similar medida. El promedio de la frecuencia cardiaca refleja las diferencias de intensidad que adoptaron los alumnos después de la capacitación donde se obtuvo una intensidad de 16 latidos más por minuto.

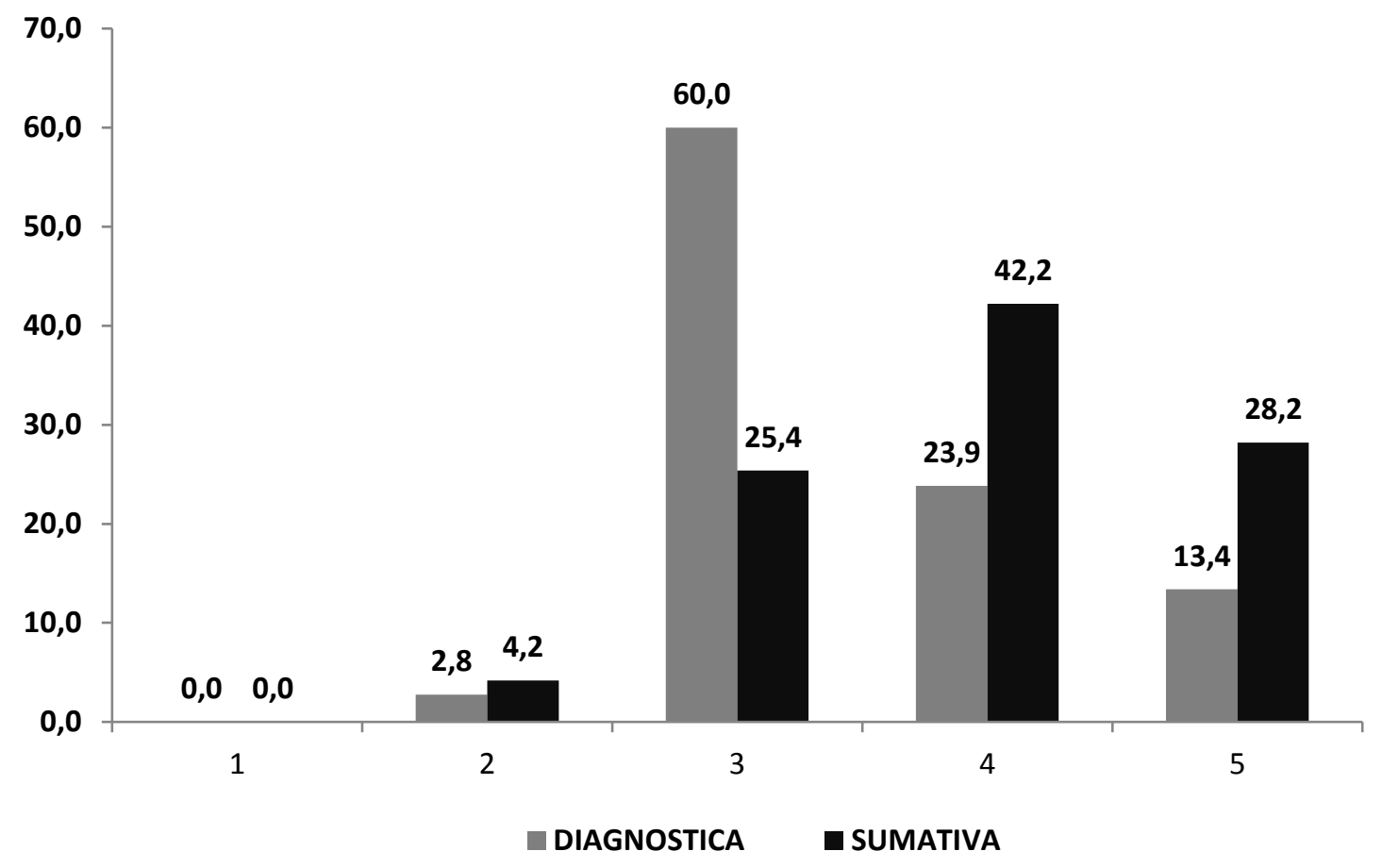

Figura 1. Distribución porcentual de la intensidad de actividad física de antes y después de la capacitación. 


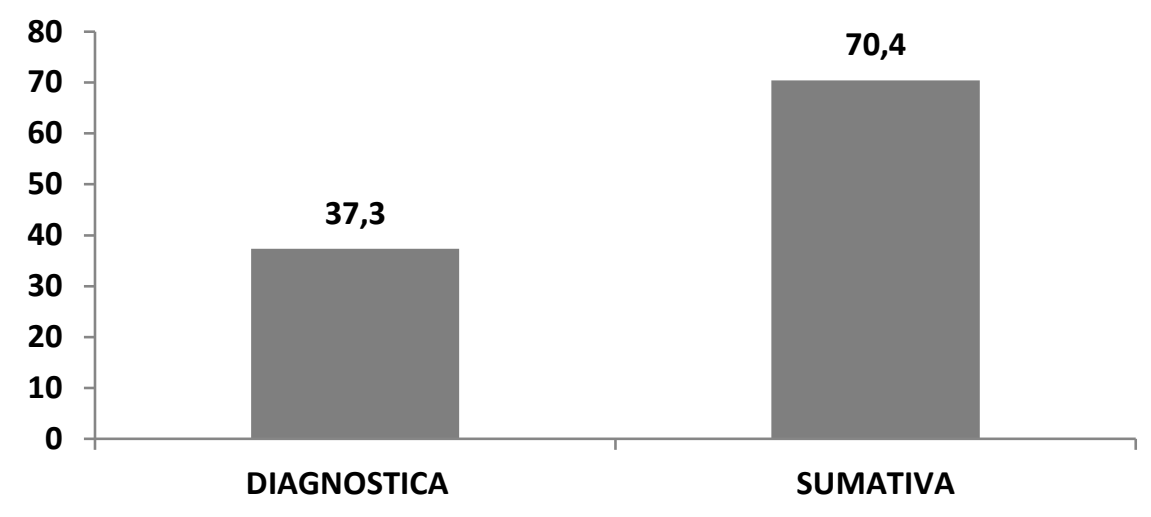

- ÍNDICE DE ACTIVIDAD FÍSICA MODERADA A VIGOROSA

Figura 2. Distribución porcentual del índice de actividad física moderada a vigorosa antes y después de la capacitación.

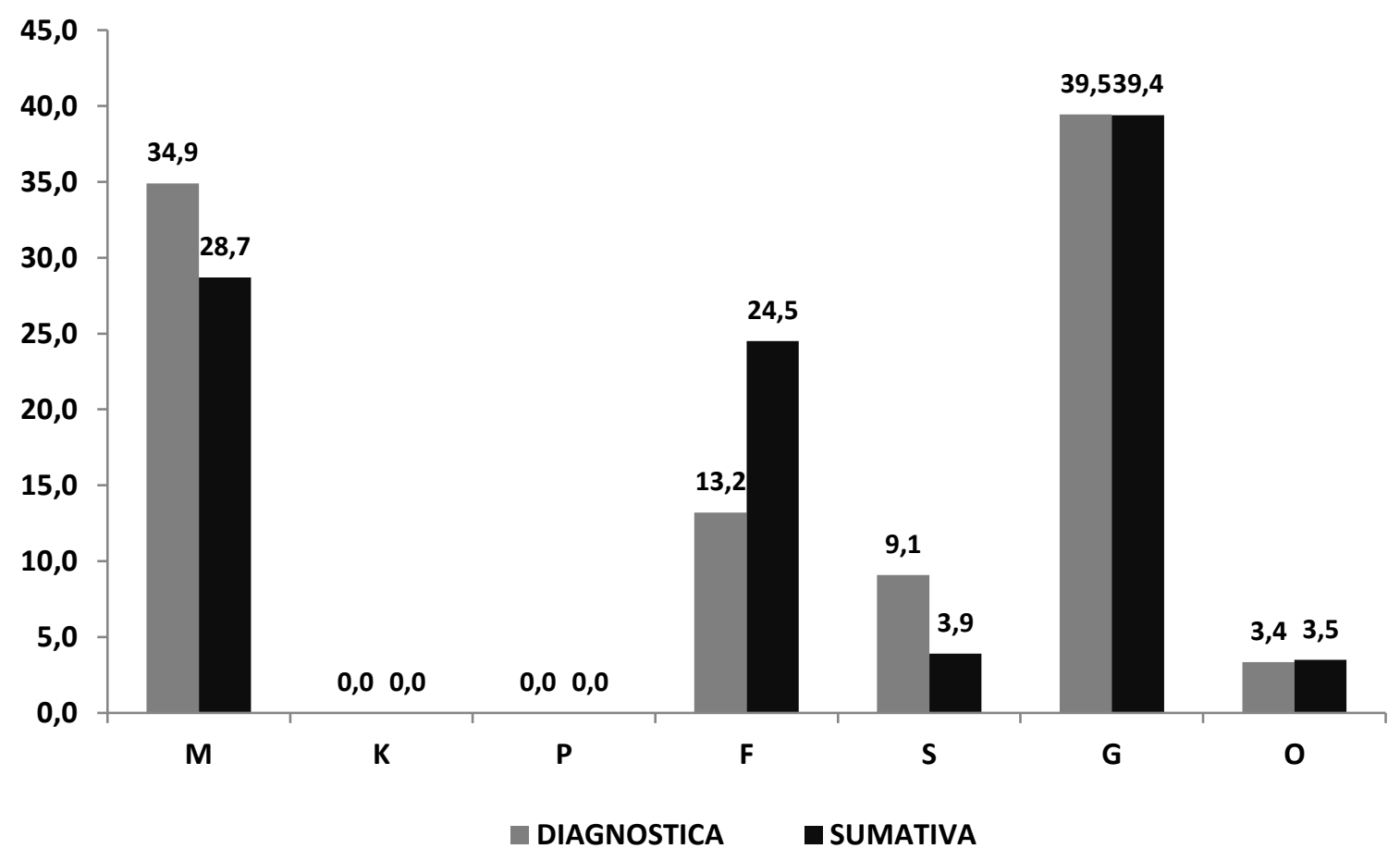

Figura 3. Distribución porcentual del contexto de la educación física antes y después de la capacitación. 


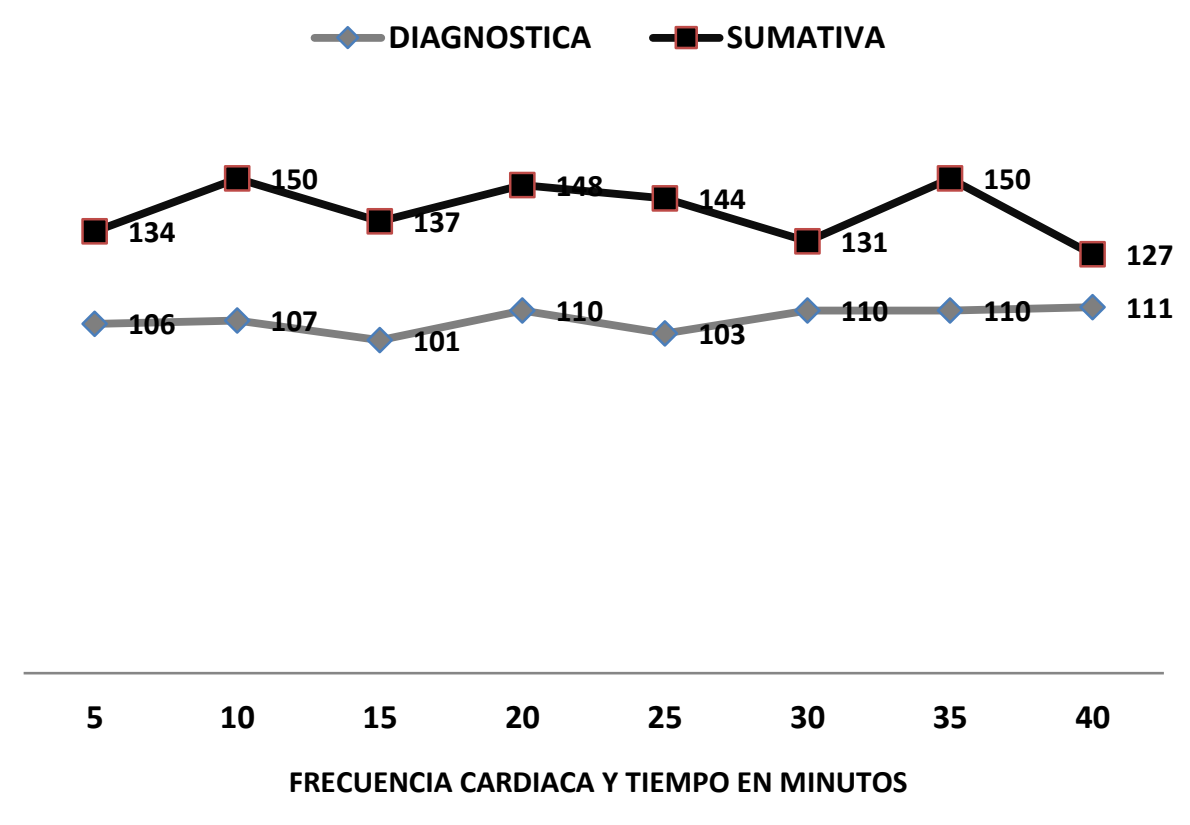

Figura 4. Frecuencia cardiaca promedio tomada cada cinco minutos antes y después de la capacitación

\section{DISCUSIÓN Y CONCLUSIONES}

Previo a la capacitación la intensidad de actividad física moderada a vigorosa de las sesiones de la clase de EF evaluadas, se cuantificó los niveles 4 y 5 representando el $37.3 \%$ del tiempo de la clase, presentándose inadecuado de acuerdo a los estándares establecidos por el United States' National Association for Sport and Physical Education (NASPE). Después de la capacitación se plantearon estrategias en las cuales las formaciones no predominaban y las oportunidades de participar fueron más probables incrementando el movimiento en los niveles 4 y 5 de la clase de EF llegando hasta un $70.4 \%$ actividad física moderada a vigorosa de las sesiones de la clase de EF. En estudios realizados con similar metodología se reporta porcentajes de actividad física moderada a vigorosa de $38.2 \%$ (Pérez Bonilla, 2009) y 29.2\% (Jennings-Aburto et al., 2009).

En cuanto al contexto de la clase de EF en las estrategias didácticas implementadas por el profesor, se puede observar como después de la capacitación se distribuyó el contexto disminuyendo así el tiempo empleado para administrar y organizar las actividades relacionadas con instrucciones tales como la formación de equipos, cambio de equipos o cambio de actividades dentro de la clase. Y se aprovecharon para acondicionamiento físico, desarrollo de habilidades y juego en intensidades de 4 y 5 , reflejándose en el incremento de la frecuencia cardiaca promedio de la clase de educación física (Santa María et al., 2011).

Con los resultados obtenidos al evaluar la clase de EF antes y después de una capacitación, en los sujetos evaluados podemos afirmar que a través del SOFIT, se pudo analizar de manera cuantitativa la intensidad y contexto de la clase impartida por 
el profesor de EF, aportando información para retroalimentar de manera constructiva la intervención pedagógica del docente de EF. Se recomienda buscar estrategias que coadyuven a maximizar el uso del tiempo de la clase de EF y que mantengan a los estudiantes en intensidades moderadas a vigorosas por lo menos el $50 \%$ de la clase utilizando contextos de administración de la clase, los cuales involucren el movimiento del alumnado.

\section{REFERENCIAS}

Ebbeling, C. B., Pawlak, D. B., y Ludwig, D. S. (2002). Childhood obesity: public-health crisis, common sense cure. Lancet, 360, 473-82.

Freedman, D. S., Dietz, W. H., Srinivasan, S. R., Berenson G. S. (1999). The relation of overweight to cardiovascular risk factors among children and adolescents: The Bogalusa Heart Study. Pediatrics, 103,1175-1182.

Hernández, B., Gortmaker, S. L., Colditz, G. A., Peterson, K. E., Laird, N. M., y ParaCabrera, S. (1999) Association of obesity with physical activity, television programs and other forms of video viewing among children in Mexico City. International Journal of Obesity and Related Metabolic Disoreder, 23, 845854.

Jennings-Aburto, N., Nava, F., Bonvecchio, A., Safdie, M., González-Casanova, I., Gust, T., y Rivera, J. (2009). Physical activity during the school day in public primary schools in Mexico City. Salud Pública México, 51(2), 141-147.

Luepker, R. V., Perry, C. L., McKinlay, S. M., Nader, P.R., Parcel, G. S., Stone, E. J., Webber, L. S., Elder, J. P., Feldman, H. A., Johnson, C. C., Kelder, S. H., y Wu, M. (1996). Outcomes of a field trial to improve children's dietary patterns and physical activity: The Child and Adolescent Trial for Cardiovascular Health (CATCH). JAMA, 275(10), 768-776.

Nader, P. R., Stone, E. J., Lytle, L. A., Perry, C. L., Osganian, S. K., Kelder, S., Webber, L. S., Elder, J. P.,Montgomery, D., Feldman, H. A., Wu, M., Johnson, C., Parcel, G., y Luepker, R.V. (1999). Three-year maintenance of improved diet and physical activity: The CATCH Cohort. Archives of Pediatrics and Adolescent Medicine, 153(7), 695-704.

National Association for Sport and Physical Education. Physical activity for children (2004). A statement of guidelines, 2nd ed. Reston, VA: National Association for Sport and Physical Education.

McKenzie, T., Sallis, J., y Nader, P. (1991). SOFIT. System for Observing Fitness Instruction Time. Journal of Teaching in Physical Education, 11, 195-205.

McKenzie, T. L., Sallis, J. F., y Armstrong, C.A. (1994). Association between direct observation and accelerometer measures of children's physical activity during physical education and recess. Medicine and Science in Sports and Exercise, 26, S143.

Montero, I., y León, O. G. (2002). Clasificación y descripción de las metodologías de investigación en Psicología. Revista Internacional de Psicología Clínica y de la Salud, 2(3), 503-508. 
Olaiz, G., Rivera, J, Cuevas, L, Shamah, T, Villalpando, S, Hernández, M, y Sepúlveda, J. (2006). Encuesta Nacional de Salud y Nutrición 2006. Estado nutricio. Cuernavaca, Morelos, México: Instituto Nacional de Salud Pública.

Pérez Bonilla, A.M. (2009) Impacto de la clase de educación física sobre la actividad moderada y vigorosa en niños de primaria. Revista Mexicana de Investigación en Cultura Física y Deporte,1(1), 150-172.

Santa María, C. J., Laíño, F. A., y Pintamalli J, C. (2011). Gasto energético en las clases de educación física de escuelas primarias y secundarias de la ciudad de buenos aires. ReCAD - Revista electrónica de Ciencias Aplicadas al Deporte, 4(12), 113.

Trost, S. G., Kerr, L. M., Ward, D. S., y Pate, R. R. (2001). Physical activity and determinants of physical activity in obese and non-obese children. International Journal of Obesity and Related Metabolic Disoreder, 25, 822-829. 